\title{
Genome-wide Characterization and Expression Analysis of YABBY Gene Family in Three Cultivars of Cucurbita Linn. And Their Response of Salt Stress in Cucurbita Moschata
}

\author{
Jingping Yuan \\ Henan Institute of Science and Technology \\ Changwei Shen ( $\nabla$ changweishen@163.com ) \\ Henan Institute of Science and Technology \\ Jingjing Xin \\ Henan Institute of Science and Technology \\ Zhenxia Li \\ Henan Institute of Science and Technology \\ Xinzheng Li \\ Henan Institute of Science and Technology \\ Junguo Zhou \\ Henan Institute of Science and Technology
}

Research article

Keywords: Cucurbita Linn., YABBY, transcriptional profiles, salt stress

Posted Date: December 8th, 2020

DOI: https://doi.org/10.21203/rs.3.rs-121953/v1

License: () (1) This work is licensed under a Creative Commons Attribution 4.0 International License. Read Full License 


\section{Abstract}

Background

Plant specific YABBY transcription factors have important biological roles in plant growth and abiotic stress. However, the identification of Cucurbita Linn. YABBY and their response to salt stress have not yet been reported. The gene number, gene distribution on chromosome, gene structure, protein conserved structure, protein motif and the cis-acting element of YABBY in three cultivars of Cucurbita Linn. were analyzed by bioinformatics tools, and their tissue expression patterns and expression profile under salt stress were analyzed.

Results

In this study, 34 YABBY genes (11 CmoYABBYs in Cucurbita moschata, 12 CmaYABBYs in Cucurbita maxima, and 11 CpeYABBYs in Cucurbita pepo) were identified and they were divided into five subfamilies (YAB1/YAB3, YAB2, INO, CRC and YAB5). YABBYs in the same subfamily usually have similar gene structures (intron-exon distribution) and conserved domains. Chromosomal localization analysis showed that these $C m o Y A B B Y s, C m a Y A B B Y s$, and CPeYABBYs were unevenly distributed in 8, 9, and 9 chromosomes of 21 chromosomes, respectively. Total of 6 duplicated gene pairs, and they all experienced segmental duplication events. Cis-acting element analysis showed that some Cucurbita Linn. YABBYs were associated with at least one of plant hormone response, plant growth, and abiotic stress response. Transcriptional profiles of $C m o Y A B B Y s$ and $C m a Y A B B Y s$ in roots, stems, leaves, and fruits, and CPeYABBYs in seed and fruit mesocarp showed that YABBYs of Cucurbita Linn. had tissue specificity. Finally, the transcriptional profile of 11 CmoYABBYs in leaf and qRT-PCR analysis of CmoYABBYs in root under salt stress indicated that some genes may play an important role in salt stress.

Conclusions

Genome-wide identification and expression analysis of YABBYs revealed the characteristics of YABBY gene family in three cultivars of Cucurbita Linn.. Transcriptome and qRT-PCR analysis revealed the response of the $C m o Y A B B Y s$ to salt stress. This provides a theoretical basis for the functional research and utilization of YABBY genes in Cucurbita Linn.

\section{Background}

Plants are usually exposed to the extreme environment during their growth and development. Salt, drought, high temperature, low temperature, and other abiotic stresses have adverse effects on the growth of plants, resulting in loss of yield and quality [1]. In plants, transcription factors (TFs) play an important role in regulating gene expression of the plant defense system, and some of them are involved in abiotic stress response [2]. So far, there are about 30 common TFs, about half of which are deemed to be plant-specific TF families, such as AP2/ERF, WRKY, NAC, B3, SBP, and DOF families [3].

YABBY is a unique transcription factor in plants, belonging to the zinc finger protein superfamily. YABBY protein consists of two highly conserved domains: $\mathrm{N}$ terminal C2C2 zinc finger motif and C-terminal YABBY domain [4-6]. Previous studies have shown that some zinc fingers have been observed as DNA-binding motifs, while many of them play a role in protein-protein interactions rather than binding to DNA [4].

YABBY transcription factors have been widely investigated in dicotyledons. There are six $Y A B B Y$ members in Arabidopsis thaliana, $Y A B B Y 1$ (FIL, filamentous lower), YABBY2 (YAB2), YABBY3 (YAB3), YABBY4 (INO, inner no outer), YABBY5(YAB5) and CRC (crabs claw). The main function of the YABBY in Arabidopsis thaliana is to specify the distal cell fate of lateral organs [7]. The evolutionary relationship has shown that $Y A B B Y s$ have different functions, including processes of plant growth and development, such as controlling the carpel number of tomato flowers [8]. Besides, $Y A B B Y$ is also affected by auxin, GA, and other hormones [9].

It is worth noting that $Y A B B Y$ plays a major role in the regulation of plant abiotic stresses, which is both an activator and an inhibitor [10]. In pineapple, RTqPCR showed that the expression of $A C Y A B B Y 2, A C Y A B B Y 3, A c Y A B B Y 6$, and $A c Y A B B Y 7$ were highly susceptible to abiotic stress. Under NaCl stress, the overexpression of $A C Y A B B Y 4$ in Arabidopsis thaliana resulted in short roots, indicating that $A c Y A B B Y 4$ plays a negative regulatory role in salt tolerance [11]. GmYABBY10 in soybean involved in high salt and drought stresses [2]. At present, although 6 YABBYs are found in Arabidopsis, 8 YABBYs are found in rice, 1 $Y A B B Y$ is found in tomatoes [12], and $Y A B B Y s$ are also found in other species. However, there isn't any previous work on role of YABBY transcription factors in modulating salt stress and other abiotic stresses in Cucurbita Linn..

The species of Cucurbita Linn. are one of the oldest crops cultivated by human beings. They have a long history, a wide range of varieties, strong adaptability, wide geographical distribution and high yield. The shape, size, and quality of the fruit are different, and the fruit color is colorful [13]. Cucurbitaceae plants are strongly affected by salt stress [14]. Under salt treatment, Cucurbitaceae plants are divided into $\mathrm{Na}^{+}$overground accumulation type and $\mathrm{Na}^{+}$underground accumulation type. The grafted cucumber seedlings with $\mathrm{Na}^{+}$underground accumulation type as rootstock could significantly reduce the content of $\mathrm{Na}^{+}$in overground and have stronger salt tolerance [15]. Three cultivars of Cucurbita Linn. (Cucurbita moschata, Cucurbita maxima, and Cucurbita pepo) are involved here. In this study, YABBY genes in three cultivars of Cucurbita Linn. were identified. Additionally, the distribution, physicochemical properties, phylogenetic relationship, structural conservation, gene duplication and tissue expression patterns of the $Y A B B Y$ gene were studied. Finally, the expression profile of Cucurbita moschata $Y A B B Y$ genes under salt stress was analyzed. The results have potential significance for the functional study and molecular mechanism of the $Y A B B Y s$ in Cucurbitaceae plants.

\section{Results}

Identification and characterization of Cucurbita Linn.YABBY transcription factor 
YABBY proteins of Cucurbita moschata, Cucurbita maxima, and Cucurbita pepo were identified by the local BLASTp program using six AtYABBY protein sequences as query sequences. All candidate protein sequences were confirmed by the NCBI CD-search. Finally, 11, 12, and 11 YABBY proteins were identified in Cucurbita moschata, Cucurbita maxima, and Cucurbita pepo, respectively. The YABBY genes were named according to the naming of the AtYABBY genes and the position on the chromosome (from the first chromosome to the last chromosome, from the top position to the end position of one chromosome) (Table 1).

The amino acid sequence of YABBY was analyzed by the ExPASy proteomics server. The results showed that the coding regions of $11 \mathrm{CmoYABBY}$ ranged from $510 \mathrm{bp}$ (CmoYAB2) to $930 \mathrm{bp}$ (CmolNOa) (Table 1). The number of translated amino acids ranged from 169 aa to 309 aa, and the $M W$ of protein ranged from 18889.49 Da to 35478.2 Da. The Pl is between 6.29 (CmoYAB5b) and 9.99 (CmoCRCb). In Cucurbita maxima, the coding regions of 12 CmaYABBY genes ranged from $537 \mathrm{bp}$ (CmaYAB5b) to $1179 \mathrm{bp}$ (CmalNOa) (Table 1). The number of translated amino acids ranged from 178 aa to 392 aa, and the $M$ W of protein ranged from 19479.12 Da to $43636.39 \mathrm{Da}$. The Plis between 6.37 (CmalNOb) and 9.32 (CmaYAB2). In Cucurbita pepo, the coding regions of 11 CpeYABBY genes ranged from $471 \mathrm{bp}$ (CpelNOb) to $1110 \mathrm{bp}$ (CpeYAB1a) (Table 1). The number of translated amino acids ranged from 156 aa to 369 aa, and the $M W$ of protein ranged from 17044.7 Da to $40999.44 \mathrm{Da}$. The $P /$ is between 6.06 (CpeYAB5a) and 9.76 (CpeYAB1b). Based on the physical and chemical characteristics of the YABBY gene in three cultivars of Cucurbita Linn., it was found that they had similar characteristics, and they had the properties of basic protein (Table 1). The predicted subcellular localization results showed that 34 Cucurbita Linn. YABBY genes were all located to the nucleus (Table 1), which accorded with the characteristics of transcription factors.

\section{Phylogenetic relationship of YABBY in three cultivars of Cucurbita Linn.}

In order to analyze the evolutionary relationship of YABBY proteins, we constructed a phylogenetic tree with 6 AtYABBYs, 11 CmoYABBYs, 12 CmaYABBYs and 11 CpeYABBYs (Fig. 1). All YABBYs were divided into five subfamilies (YAB1/YAB3, YAB2, INO, CRC and YAB5) according to the identity of amino acid sequences. Each subfamily contained AtYABBY, CmoYABBY, CmaYABBY and CpeYABBY proteins. The YAB1/YAB3 subfamily consisted of 2 CmoYABBYs, 3 CmaYABBYs, 3 CpeYABBYs, 2 AtYABBYs (AtYAB1 and AtYAB3); YAB2 subfamily contained 4 proteins: CmoYAB2, CmaYAB2, CpeYAB2 and AtYAB2; YAB5 subfamily contained 4 CmoYABBYs, 4 CmaYABBYs, 4 CpeYABBYs and AtYAB5; INO subfamily contained 2 CmoYABBYs, 2 CmaYABBYs, 2 CpeYABBYs and AtINO; CRC subfamily contained 2 CmoYABBYs, 2 CmaYABBYs, 1 CpeYABBYs and AtCRC proteins.

\section{Gene structures and motif composition of YABBY gene family in three cultivars of Cucurbita Linn.}

The gene structure can provide valuable information for the phylogenetic relationship of YABBY in three cultivars of Cucurbita Linn.. Based on the phylogenetic tree, the exon-intron structure and conserved motifs of 34 YABBYs were analyzed by TBtools (Fig. 2B). The classification pattern of exon-intron was consistent with the phylogenetic tree (Fig. 1; Fig. 2A). All YABBYs from three cultivars of Cucurbita Linn. contain introns, and the number of exons was 512. The number of exons and the length of introns in one branch were similar. For instance, in the YAB2 branch, all $Y A B B Y$ genes contained six exons. What's more, from the phylogenetic analysis, the closer the homology was, the more similar the structure was, such as CmoYAB1a_CmaYAB1a,

CmoYAB5b_CmaYAB5b, and CmoYAB1b_CmaYAB1b (Fig. 2B). Through multiple sequence alignment, it was found that YABBY protein contained a conserved YABBY domain at the C-terminal, and most YABBY proteins contained a conserved C2C2 domain (Fig. S1). Ten conserved motifs of YABBYs were searched and identified by MEME online tools (Fig. 2C; Fig. S2). Motif analysis showed that motifs 1 and 2 existed in all YABBYs proteins. In addition to CpeYAB1b protein, motif 3 existed in all other proteins. Motif 4 existed in all other proteins except CpeYAB5d. These results indicated that the domain and motif of YABBYs were highly conserved. What's more, motif 6 only existed in YAB5 subfamily, motif 9 only existed in a branch of YAB5 subfamily; motif 8 only existed in some genes of INO and YAB5 subfamily, and motif 10 only existed in some genes of YAB5 and CRC subfamily (Fig. 2C), indicating that these genes may have special functions.

Distribution, gene duplication and collinearity of YABBY transcription factors in three cultivars of Cucurbita Linn.

According to the genome sequence of Cucurbita, the chromosome position of YABBY in each cultivar of Cucurbita Linn. was determined (Fig. 3A). Eleven CmoYABBYs were located on 8 of the 21 chromosomes, and there were 2 CmoYABBYs on chromosomes Cmo_chr02, Cmo_chr04, and Cmo_chr05,

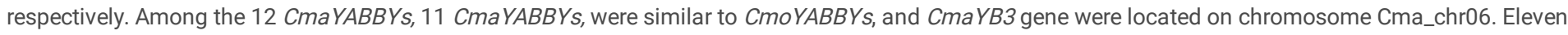
CpeYABBYs were located on 9 of 21 chromosomes. Except for 2 CpeYABBYs on chromosomes CP4.1LG05 and CP4.1LG11, respectively, there was one CpeYABBYs on the other chromosomes (CP4.1LG01, CP4.1LG04, CP4.1LG08, CP4.1LG09, CP4.1LG13, and CP4.1LG18), respectively (Fig. 3A).

According to the amino acid sequence identity $>80 \%$ and gene alignment coverage $>0.75$, we found three duplicated gene pairs (CmoYAB1a-CmoYAB1b; CmoYAB5a-CmoYAB5d and CmoYAB5c-CmoYAB5b) in CmoYABBYs, two duplicated gene pairs (CmaYAB1b-CmaYAB1a and CmaYAB5d-CmaYAB5a) in CmaYABBYs, and one duplicated gene pairs (CpeYAB5b-CpeYAB5c) in $C p e Y A B B Y s$, with all $\mathrm{Ka} / \mathrm{Ks}<1.0$, indicating that these duplicated gene pairs mainly underwent purification selection, and the divergence time was 6.64 16.47 (MYA) (Table 2).

Synteny relationship of $Y A B B Y$ genes among Arabidopsis and three cultivars of Cucurbita Linn. was also analyzed. Eight, eight and six collinear genes of AtYABBYs were found in Cucurbita moschata,Cucurbita maxima and Cucurbita pepo, respectively (Fig. 3B). Based on the collinearity analysis of YABBYs among Cucurbita moschata, Cucurbita maxima, and Cucurbita pepo, it was found that there were 30 pairs of collinear genes between CmoYABBYs and CpeYABBYs, 29 pairs of collinear genes between CmoYABBYs and CmaYABBYs and 26 pairs of collinear genes between CmaYABBYs and CpeYABBYs (Fig. 3B; Table S1).

\section{Cis-acting elements in Cucurbita Linn. YABBY gene promoters}

To understand the transcriptional regulation of the $Y A B B Y$ s in three cultivars of Cucurbita Linn., we extracted the $2.0 \mathrm{~kb}$ sequence before the translation initiation site (ATG) and predicted the cis-elements on the PlantCARE server. Fig. 4A showed the position of the cis-acting element on the promoter. It is worth 
noting that five kinds of plant hormone-responsive cis-acting elements were identified, including abscisic acid response element, methyl jasmonate response element, gibberellin response element, salicylic acid response element and auxin response element (Fig. 4A; Fig. 4B). Thirty-four YABBY gene promoters contained at least two plant hormone response element. CpelNOb and CmalNOb contained the most (21) plant hormone response elements. Among them, there were 10 and 12 elements involved in methyl jasmonate, respectively (Fig. 4B). Additionally, we found that 74\% (25) of the YABBYS contain elements that participate in the growth and development of plants, including 19 genes involved in meristem expression, and 3 genes (CmoYAB1b, CmaYAB $1 b$, and $C p e Y A B 1 b$ ) involved in seed-specific regulation (Fig. 4C). The $Y A B B Y$ s may also respond to abiotic stresses such as defense and stress, anaerobic, droughtinduced and low temperature (Fig. 4D). CmalNOb contained the highest number (9) of abiotic stress elements, and CmoYAB5a contained 5 elements involved in anaerobic induction. These data suggestted that $Y A B B Y$ may be involved in plant hormone response, abiotic stress, and plant growth and development through a complex mechanism.

\section{The expression profiles of CmoYABBYs and CmaYABBYs in different tissues}

According to RNA-seq data (BioProject: PRJNA385310), the expression profiles of CmoYABBYs and CmaYABBYs in root, stem, leaf and fruit were obtained. In general, CmoYABBY and CmaYABBY genes were mainly expressed in roots and leaves (Fig. 5). In Cucurbita maxima, CmoYAB1a, CmoYAB5a, CmoYAB5a, and CmoYAB5a were mainly expressed in leaves. The expression of $C m o Y A B 1 b$ in the root, leaf, and fruit was higher than that in the stem. The relative expression of CmoINOa in four tissues was higher than other genes. CmoYAB2 was mainly highly expressed in root and stem; The remaining $C m o Y A B B Y$ genes have a low expression (Fig. 5A). In Cucurbita maxima, CmaYAB1b, CmaYAB5a, CmaYAB3, CmaINOa, CmaYAB5c, and CmaYAB1a were mainly expressed in leaves. The expression of $C m a Y A B 2$ in stems, leaves, and fruits was higher than that in roots. CmaYAB5b was highly expressed in all tissues. The relative expression levels of other CmaYABBY genes were relatively low (Fig. 5B). Based on the above analysis, we speculated that $C m o Y A B B Y s$ and $C m a Y A B B Y s$ have tissue specificity.

\section{Expression profiles of CpeYABBYs in fruit mesocarp and seed}

To study the expression patterns of CPeYABBYs in seed and fruit mesocarp, the Cucurbita pepo cultivar, "Sweet REBA" was used and the published transcriptome data (BioProject: PRJNA339848) [16] was naalysed. It showed that all CpeYABBYs (except CpeCRC) were highly expressed in the seed at 20 days after pollination (DAP). Meanwhile, CPeYAB5c, CpeYAB5a, CpeYAB1a, CpeYAB1b, CpelNOa, CpelNOb and CpeYAB2 were highly expressed in the fruit mesocarp at 20 days after pollination (DAP). CPeYAB2 had a relatively high relative expression profile at each developmental stage in both seed and fruit mesocarp (Fig. 6).

\section{Expression patterns of CmoYABBYs in leaf mesophyll and leaf vein under salt stress}

To determine the expression pattern of the YABBY gene in Cucurbita moschata (salt-sensitive type) under salt stress, the RNA-seq data (BioProject: PRJNA464060) was analyzed. The results showed that the expression of all CmoYABBYs (except for CmoCRCa was unchanged) in leaf veins was inhibited under salt stress, while all $C m o Y A B B Y s$ in leaf mesophyll were induced. For instance, the expression level of $C m o Y A B 1 a$ in the leaf vein under salt stress was significantly reduced compared with the control treatment, while the relative expression of CmoYAB1a in leaf mesophyll under salt stress was 2.29 times higher than the control treatment (Fig. 7).

\section{Expression patterns of CmoYABBYs in root tip under salt stress}

Tissue location analysis showed that all CmoYABBYs were mainly expressed in roots and leaves (Fig. 5A). In the leaves, the expression level of $C m o Y A B B Y s$ in the leaf vein was significantly inhibited under salt stress, while the expression level of $C m o Y A B B Y s$ in the leaf mesophyll was significantly induced under salt stress. However, the response of $C m O Y A B B Y$ in the root to salt stress was not clear. For further determine the response of $C m o Y A B B Y s$ in root to salt stress, the qRT-PCR data was analyzed. The results showed that most of the CmoYABBYs had low expression levels in root tips, but $C m o C R C b, C m o l N O b$ and $C m o Y A B 5 d$ were significantly induced under salt stress. For example, the relative expression profile of CmolNOb was 6.45 times that of the control. Therefore, we speculated that $\mathrm{CmolNOb}$ in root tips may play an important role in salt tolerance.

\section{Discussion}

Plant specific YABBY transcription factors play important biological roles in plant morphogenesis, growth and development, and abiotic stress response [17]. However, only a few species of $Y A B B Y$ gene family have been identified at the genome level, such as tomato [12], pineapple [11], soybean [2], grape [18] and cotton [19]. As far as we know, there was no systematic report on the YABBY gene family in Cucurbita Linn.. In this study, we explored 34 YABBY genes in three cultivars of Cucurbita Linn. by analyzing the phylogeny, chromosomal distribution, gene structure, conserved motifs, and cis-acting elements.

Based on phylogenetic tree analysis of YABBY proteins in three cultivars of Cucurbita Linn., it was found that they were divided into five subfamilies (YAB1/YAB3■YAB2 INOICRC and YAB5), which was consistent with the previous classification of AtYABBYs [17]. Besides, phylogenetic tree analysis found that each subfamily contains $C m o Y A B B Y, C m a Y A B B Y$, and $C P e Y A B B Y$, indicating that they may have evolved from the same ancestor. In Arabidopsis thaliana, AtFIL, AtYAB2 and $A t Y A B 3$ are expressed in the adaxial region of all lateral organs, including cotyledons, leaves and flowers. However, AtINO is limited to the integument of ovule [20,7]. Phylogenetic analysis showed that $A t Y A B 1(\mathrm{FIL})$ and $A t Y A B 3$ had the closest homology with $C m a Y A B 3$ and $C p e Y A B 3$, $A t Y A B 2$ had the closest homology with CmoYAB2. Therefore, we speculated that CmaYAB3, CpeYAB3 and CmoYAB2 might participate in the expression of adaxial region in lateral organs. Besides, we found that $A$ tINO had the closer homology with CmoINOb, CmaINOb and CpeINO than other genes. Therefore, it is speculated that they play an important role in the integument of ovule. AtCRC participates in flower development [21], so the homologous genes $C m a C R C b$, CmaCRCb may have the same function as AtCRC. 
Through the analysis of $Y A B B Y$ gene structure, it is found that most genes in the same subfamily have similar structural characteristics in terms of the number of exons or introns, which is similar to the structural characteristics of the YABBYs in other species [19, 22]. However, CmaINOa and CpeYAB1a have a different number of exons compared with other $Y A B B Y$ s in the same subfamily, which indicates the structural diversity of the $Y A B B Y$ gene family in $C u c u r b i t a$ Linn.. For a specific motif, high differences were also detected among different subfamilies. However, in one subfamily, most YABBY proteins of Cucurbita Linn. have conserved motifs, which suggests that the same subfamily may have similar functions.

Gene duplication plays an important role in biological evolution, including fragment duplication, tandem duplication and whole genome duplication [23]. Cucurbita experienced Cucurbit-common tetraploidization (CCT) events[13], and Cucurbita shared a recent Cucurbita-specific tetraploidization (CST) [24]. Six duplicated gene pairs were found in three cultivars of Cucurbita Linn.. Because these duplicated gene pairs were not on the same chromosome, we termed them fragment duplicated gene pairs. Ka / Ks $<1$ indicated that these $Y A B B Y$ genes were in the process of purification and positive selection. The detailed analysis of collinear gene pairs and evolutionary relationship revealed the high complexity of chromosome evolution and rearrangement of three Cucurbita Linn. cultivars. Therefore, it is not surprising that the $Y A B B Y$ genes in Cucurbita moschata, Cucurbita maxima, and Cucurbita pepo are highly similar, and these $Y A B B Y$ genes may have similar functions.

Cis-acting elements can be bind specifically by transcription factors and can regulate gene transcription [25]. At least two cis-acting elements related to plant hormone response were found in $34 Y A B B Y$ gene promoters, which means that the corresponding hormones may play an important role in its regulation. Besides, all $Y A B B Y$ genes (except for CmaYAB2) contain at least one cis-acting element related to stress response (Fig. 4C), indicating that these genes also play an important regulatory role in stress response. However, further research is needed to determine whether and how these cis-acting elements work in Cucurbita Linn.

The response of $Y A B B Y s$ in "Rifu" to salt stress showed that there was differential expression of $Y A B B Y$ in leaf mesophyll and leaf vein of Cucurbita moschata under salt stress. The expression of CmoYABB1a was inhibited in leaf veins under salt stress, and induced in leaf mesophyll, which may have an important relationship with sodium ion effloression of pumpkin under salt stress (Fig. 7). High concentration of $\mathrm{Na}^{+}$in plants has a strong toxic effect on leaf photosynthetic organs. Under salt treatment, Cucurbita moschata were divided into $\mathrm{Na}^{+}$above-ground accumulation type and $\mathrm{Na}^{+}$underground accumulation type, and grafted cucumber seedlings with $\mathrm{Na}^{+}$underground accumulation type as rootstock could significantly reduce the content of $\mathrm{Na}^{+}$in aboveground and have stronger salt tolerance [15]. The main reason is that Cucurbita moschata rootstocks have strong $\mathrm{Na}^{+}$storage capacity and strong $\mathrm{Na}^{+}$root effluents, which further reduces the transport of $\mathrm{Na}^{+}$to the overland part and the content of $\mathrm{Na}^{+}$in the scion leaves [26]. To further verify the response of CmoYABBYs to salt stress, we also analyzed the root tips of Cucurbita moschata "Baimi 9". It was found that CmolNOb may play a key role in salt stress (Fig. 8), and the $Y A B B Y$ gene family of Cucurbita was may be involved in the response to salt stress.

\section{Conclusions}

In summary, we identified 34 YABBYs in three cultivars of Cucurbita Linn. based on a thorough analysis and provided genetic information such as chromosome locations and exon-intron structures, conserved domains, and duplicated genes. We specifically examined the expression profiles of these $Y A B B Y s$ in different tissues. At the same time, we examined the responses of CmoYABBYs to salt stress, and several key genes were found to regulate the resistance of three of Cucurbita Linn. cultivars.

\section{Methods}

\section{Identification and characterization of Cucurbita Linn. YABBY transcription factor}

Six Arabidopsis thaliana YABBYs (AtYABBYs) protein sequences from PlantTFDB database (http://planttfdb.cbi.pku.edu.cn/download_seq.php?Fam=YABBY) were used as query objects to conduct local BLAST [27] (E-value $<1 \mathrm{e}^{-5}$, identity $\left.>50 \%\right)$ ) on the genome databases of Cucurbita moschata, Cucurbita maxima and Cucurbita pepo (http://cucurbitgenomics.org/). The HMM model of the YABBY domain (PF04690) in the Cucurbitaceae protein database was analyzed by using HMMER 3.0 software $\left(E-v a l u e<1 \mathrm{e}^{-5}\right)$ [28]. Also, all YABBY protein sequences obtained were further analyzed on CDD

(https://www.ncbi.nlm.nih.gov/cdd) [29] to verify whether C2C2 domain exists in the N-terminal and YABBY domain in C-terminal.

Using the ExPASY server (https://web.expasy.org/protparam/) to predict the physical and chemical properties of the YABBY protein, including the length of an amino acid sequence, molecular weight $(M W)$, and isoelectric point $(P I)$. The subcellular localization of YABBYs in three cultivars of Cucurbita Linn. was predicted by the Plant-mPLoc server (http://www.csbio.sjtu.edu.cn/bioinf/plant-multi/\#) [30]. To obtain the location information of $Y A B B Y s$ in three cultivars of Cucurbita Linn., the starting position, end position, and chromosome length of $Y A B B Y s$ were obtained from the Cucurbita database, and the distribution map of YABBYs was drawn by TBtools [31].

\section{Structure analysis of Cucurbita Linn. YABBYs}

The cDNA and DNA sequence of $Y A B B Y s$ were obtained from the Cucurbita database. The exon-intron structural map of $Y A B B Y S$ was drawn by TBtools software [31]. All YABBY protein sequences in three cultivars of Cucurbita Linn. were aligned with DNAMAN software and corrected manually [32]. At the same time, the online software MEME (http://meme.nbcr.net/meme/cgi-bin/meme.cgi) [33] was utilized to predict the conserved motifs. The parameter was set as the number of motifs: 10 , the length of motifs: 5-50.

\section{Construction of phylogenetic tree}


All Cucurbita Linn. YABBY proteins were aligned by ClustalW. The phylogenetic relationship between AtYABBY proteins and YABBY protein from three cultivars of Cucurbita Linn. was constructed by MEGA 7.0 [34] using the neighbor-joining ( $\mathrm{NJ}$ ) method. The parameters were as follows: completed deletion, poisson model, cut off value for the condensed tree was $65 \%$.

\section{Collinearity and gene duplicated of YABBY in Cucurbita Linn.}

The collinear genes of AtYABBYs in three Cucurbita cultivars were obtained by TBtools. Finally, the collinear relationship of YABBYS was drawn by Circos [35].

The CDS of $Y A B B Y S$ were blasted by the TBtools program, and the gene alignment coverage of $Y A B B Y s$ was calculated by the formula: gene alignment coverage $=($ alignment length - mismatches $)$ /length of larger genes. The duplication of gene pairs is performed according to the previous indicators (the amino acid identity $>80 \%$, the E-value $<1 \times 10^{-10}$ and the gene alignment coverage $>0.75$ ) in Chinese cabbage [36]. Besides, when the interval between the two genes is less than $100 \mathrm{~kb}$, it is considered to be a tandem duplicated gene [37]. The synonymous substitution rate $\left(K_{S}\right)$ of $Y A B B Y$ gene was calculated by $K a K S$ calculator, and the divergence time (T) of $Y A B B Y$ gene was calculated according to the formula: $T=\mathrm{K}_{\mathrm{S}} / 2 \lambda \times 10^{-6}$ (MYA, where " $\lambda$ " is the neutral substitution rate, $\lambda=1.5 \times 10^{-8}[38]$.

\section{Extraction of $Y A B B Y$ promoter sequence and analysis of cis-acting elements}

To obtain the promoter sequence of the YABBY in three cultivars of Cucurbita Linn., the 2000 bp before the start codon of $Y A B B Y S$ was obtained by TBtools [31]. On this basis, the cis-acting elements of the promoters of all genes were predicted by PLantCARE

(http://bioinformatics.psb.ugent.be/webtools/plantcare/html/), and finally, use TBtools to visualize the cis-acting elements.

\section{Expression profile of Cucurbita Linn.YABBYs in RNA-Seq}

To study the expression pattern of CmoYABBYs and CmaYABBYs in different organs, we downloaded the published transcriptome data (BioProject: PRJNA385310) [39]. Besides, to study the expression pattern of $C p e Y A B B Y s$ in seed and fruit mesocarp, we downloaded the published transcriptome data (BioProject: PRJNA339848) [16].

To determine the response of Cucurbita moschata YABBY gene family to salt stress, we analyzed the expression level of the Cucurbita moschata cultivar, "Rifu" in leaf vein and leaf mesophyll under salt and control treatments. We excavated the transcriptome data (BioProject: PRJNA464060) published in 2019 [40] and analyzed the transcription profile of $Y A B B Y s$ in the leaf vein and leaf mesophyll under salt stress.

All the published transcriptome data were represented by RPKM (Reads per kilobase of exon model per million mapped reads), which has been converted to $\log _{2}$ (RPKM) when plotting heat map.

\section{Experimental materials and stress treatment}

To further clarify the differential expression of these genes in root, the Cucurbita moschata variety "Baimi 9" was used as the study material and the qRT-PCR was performed. Variety "Baimi 9" was provided by the pumpkin team of School of Horticulture and Landscape Architecture, Henan Institute of Science and Technology. The seeds were sown in a tray containing a matrix-meteorite (3:1) mixture and grown in a plant growth chamber. The artificial growth conditions were set to $25^{\circ} \mathrm{C} / 16^{\circ} \mathrm{C}, 16 \mathrm{~h}$ light / $8 \mathrm{~h}$ dark and $65 \%$ relative humidity. The two-month-old seedlings were cultured in $1 / 2 \mathrm{Hoagland}$ solution, pH 6.5 . After 5 days of adaptation, some of the seedlings were cultured with $75 \mathrm{mM} \mathrm{NaCl}$. Root tips were collected at $24 \mathrm{~h}$ after the $\mathrm{NaCl}$ treatment. Three independent biological replications formed one sample. Control and salt-treated samples were frozen in liquid nitrogen and stored at $-70{ }^{\circ} \mathrm{C}$ for further analysis.

\section{Quantitative real-time PCR (qRT-PCR) analysis}

The total RNA was extracted with RNA-Solv@ reagent (Omega), and the first strand cDNA was prepared with PrimeScript ${ }^{\mathrm{TM}}$ RT Master Mix (TaKaRa). Using CDNA as a template, the expression of $Y A B B Y$ in Cucurbita moschata root tip under salt stress was analyzed. The specific quantitative primers (Table S2) were design by Primer-BLAST program (https://www.ncbi.nlm.nih.gov/tools/primer-blast/index.cgi). The pumpkin $\beta$-Actin was used as the internal reference gene. The reaction system was $20 \mu \mathrm{L}$, including $10 \mu \mathrm{L}$ of SYBR Green I, $0.4 \mu \mathrm{L}$ of ROX dye II, $0.4 \mu \mathrm{L}$ of primers, $2 \mu \mathrm{L}$ of cDNA template and $6.8 \mu \mathrm{L}$ of dd $\mathrm{H}_{2} \mathrm{O}$. PCR was performed on Applied Biosystems 7500 , and the reaction conditions were set as follows: $95^{\circ} \mathrm{C}$ pre-denaturation for $30 \mathrm{~s}$, $95^{\circ} \mathrm{C}$ denaturation for $5 \mathrm{~s}, 60$ ${ }^{\circ} \mathrm{C}$ degradation and extension for $34 \mathrm{~s}$ and 40 cycles. The melting curve is: $95^{\circ} \mathrm{C}$ for $15 \mathrm{~s}, 60^{\circ} \mathrm{C}$ for $60 \mathrm{~s}, 95^{\circ} \mathrm{C}$ for $15 \mathrm{~s}$. The $2^{-\Delta \Delta c t}$ method $[41]$ was used to quantitatively analyze the data. Each reaction was performed three times, and the data was presented by heatmap.

\section{Abbreviations}

TF: Transcription factor; qRT-PCR: Quantitative real-time polymerase chain reaction; MW: Molecular weight; pl: Isoelectric point; NJ: Neighbor-joining; GSDS: Gene structure display server; MEME: Multiple Expectation Maximization or Motif Elicitation; Ks:Synonymous substitution ratio; Mya: Million years ago; RPKM: Reads per kilobase of exon model per million mapped reads.

\section{Declarations}

\section{Acknowledgements}

Not applicable. 


\section{Author's contributions}

CWS conceived, designed and analyzed data; CWS and JPY wrote the manuscript; JJX and ZXL identified Cucurbita Linn. YABBYs and analyzed gene structure. XZL and JGZ studied chromosome distribution, gene duplication and syntenic analysis of Cucurbita Linn. YABBYs. JPY supervised the research. CWS and JPY revised the manuscript. All authors read and approved the manuscript.

\section{Funding}

This work was supported by the Scientific Research Foundation for High - level Talent (103010620001/015 and 2017034).

\section{Availability of data and materials}

The information on gene and protein sequence of Cucurbita moschata YABBY s was downloaded from the Cucurbit genomics database (CuGenDB, http://cucurbitgenomics.org/). The data and materials supporting our research findings were contained in the methods and additional files.

\section{Ethics approval and consent to participate}

Not applicable.

\section{Consent for publication}

Not applicable.

\section{Competing interests}

There have no competing interests among authors

\section{Author details}

${ }^{1}$ School of Horticulture and Landscape Architecture, Henan Institute of Science and Technology, Xinxiang, 453003, China. 2Henan Engineering Research Center of the Development and Utilization of Characteristic Horticultural Plants, Xinxiang, 453003, China. ${ }^{3}$ School of Resources and Environmental Sciences, Henan Institute of Science and Technology, Xinxiang 453003, China.

\section{References}

1. Zhou J Studies on screening and physiological characterstics of salt-tolerant root stocks from Cucurbita moschata Duch. Germplasm, Nanjing Agricultural University. 2008(In Chinese).

2. Zhao SP, Lu D, Yu TF, Ji YJ, Zheng WJ, Zhang SX, et al. Genome-wide analysis of the YABBY family in soybean and functional identification of GmYABBY10 involvement in high salt and drought stresses. Plant Physiol Biochem. 2017;119:132146.

3. Yamasaki K, Kigawa T, Seki M, Shinozaki K, Yokoyama S. DNA-binding domains of plant-specific transcription factors: structure, function, and evolution. Trends Plant Sci. 2013;18:267-

4. Golz JF, Roccaro M, Kuzoff R, Hudson A. GRAMINIFOLIZ promotes growth and polarity of antirrhinum leaves. Development. 2004;131:3661-

5. Sieber P, Petrascheck M, Barberis A, Schneitz K. Organ polarity in Arabidopsis NOZZLE physically interacts with members of the YABBY family. Plant Physiol. 2004;135:2172-

6. Mackay JP, Crossley M. Zinc fingers are sticking together. Trends Biochem Sci. 1998;23:1-4

7. Villanueva JM, Broadhvest J, Hauser BA, Meister RJ, Schneitz K, Gasser CS. INNER NO OUTER regulates abaxial- adaxial patterning in Arabidopsis ovules. Genes. 1999;13:3160-3169.

8. Cong B, Barrero LS, Tanksley SD. Regulatory change in YABBY-like transcription factor led to evolution of extreme fruit size during tomato domestication. Nat Genet. 2008;40:800-804.

9. Peng X, Wu Q, Teng L, Tang F, Pi Z, Shen S. Transcriptional regulation of the paper mulberry under cold stress as revealed by a comprehensive analysis of transcription factors. BMC Plant Biol. 2015;15:108. doi: 10.1186/s12870-015-0489-2

10. Schluttenhofer C, Yuan L. Regulation of specialized metabolism by WRKY transcription factors. Plant Physiol. 2015;167:295-306.

11. Li ZY, Li G, Cai MX, Priyadarshani SVGN, Aslam M, Zhou Q, et al. Genome-wide analysis of the YABBY transcription factor family in pineapple and functional identification of AcYABBY4 involvement in salt stress. Int J Mol Sci. 2019;20:1-17.

12. Huang Z, Van Houten J, Gonzalez G, Xiao H, van der Knaap E. Genome-wide identification, phylogeny and expression analysis of SUN, OFP and YABBY gene family in tomato. Mol Genet Genom. 2013;288:111-129.

13. Wang JP, Sun PC, Li YX, Liu YZ, Yang NS, Yu JG, et al. An overlooked paleotetraploidization in Cucurbitaceae. Mol Biol Evol. 2018;35:16-26.

14. Sun JY. The mechanism of CmHKT1;1 increasing salt tolerance of grafted cucumber seeding and identification of related microRNAs. Huazhong Agricultural University. 2019. (In Chinese).

15. Sun JY, Cao HS, Cheng JT, He XM, Sohail H, Niu ML, et al. Pumpkin CmHKT1;1 controls shoot $\mathrm{Na}^{+}$accumulation via limiting Na ${ }^{+}$transport from rootstock to scion in grafted cucumber. Int J Mol Sci. 2018;19(9):2648.

16. Wyatt LE, Strickler SR, Mueller LA, Mazourek M. Comparative analysis of Cucurbita pepo metabolism throughout fruit development in acorn squash and oilseed pumpkin. Hortic Res.2016;3: 
17. Zhao YJ, Liu CY, Ge DP, Yan M. Genome-wide identification and expression of YABBY genes family during flower development in Punica granatum L. Gene. 2020;752:

18. Zhang SL, Wang L, Sun XM, Li YD. Genome-wide analysis of the YABBY gene family in grapevine and functional characterization of VvYABBY4. Front Plant 2019;10:1207.

19. Yang ZE, Gong Q, Wang LL, Jin YY, Xi JP, Li Z, et al. Genome-wide study of YABBY genes in upland cotton and their expression patterns under different stresses. Front Genet. 2018;9:

20. Siegfried KR, Eshed Y, Baum SF, Otsuga D, Drews GN, Bowman JL. Members of the YABBY gene family specify abaxial cell fate in Arabidopsis. Development. 1999;126:4117-4128.

21. Sawa S, Watanabe K, Goto K, Kanaya E, Morita EH, Okada K. FILAMENTOUS FLOWER, a meristem and organ identity gene of Arabidopsis, encodes a protein with a zinc finger and HMG-related domains. Genes Dev. 1999;13:1079-1088.

22. Li XB, Yang CC, Qiu NW. Bioinformatic analysis of YABBY protein family in Arabidopsis and Chinese Cabbage. Shandong Agric Sci. 2012;44:1-6 (In Chinese).

23. Xu GX, Guo CC, Shan HY, Kong HZ. Divergence of duplicate genes in exon-intron structure. Proc Nat Acad Sci USA. 2012;109:1187-1192.

24. Yu JG, Wang JY, Wang JY, Wang Comparative genomic analysis of pumpkin, grape and wax gourd. Mol Plant Breeding. 2020;1-16 (In Chinese).

25. Riechmann JL, Heard J, Martin G, Reuber L, Jiang C, Keddie J, et al. Arabidopsis transcription factors: genome wide comparative analysis among eukaryotes. Science. 2000;290:2105-2110.

26. Huang Y, Bie ZL, He SP, Hua B, Zhen A, Liu ZX. Improving cucumber tolerance to major nutrients induced salinity by grafting onto Cucurbita ficifolia. Environ Exp Bot. 2010;69(1):32-38.

27. Camacho C, Coulouris G, Avagyan V, Ma N, Papadopoulos J, Bealer K, et al. BLAST architecture and applications. BMC Bioinformatics. 2009.

28. Mistry J, Finn RD, Eddy SR, Bateman A, Punta M. Challenges in homology search: HMMER3 and convergent evolution of coiled-coil regions. Nucleic Acids Res. 2013;41(12):

29. Marchler-Bauer A, Bo Y, Han LY, He J, Lanczycki CJ, Lu SN, et al. CDD/SPARCLE: Functional classification of proteins via subfamily domain architectures. Nucleic Acids Res. 2017;45:200-203.

30. Chou KC, Shen HB. Plant-mPLoc: a top-down strategy to augment the power for predicting plant protein subcellular localization. PloS One. 2010;5:e11335.

31. Chen CJ, Chen H, Zhang Y, Thomas HR, Frank MH, He YH, et al. TBtools - an integrative toolkit developed for interactive analyses of big biological data. Mol Plant. 2020.

32. He HH, Liang GP, Lu SX, Wang PP, Liu T, Ma ZH, et al. Genome-wide identification and expression analysis of GA20x, GA3ox, and GA20ox are related to gibberellin oxidase genes in grape (VitisVinifera). Genes (Basel). 2019;10(9):680.

33. Bailey TL, Elkan C. The value of prior knowledge in discovering motifs with MEME. Proc Int Conf Intell Syst Mol Biol. 1995;3:21-

34. Kumar S, Stecher G, Tamura K. MEGA7: molecular evolutionary genetics analysis version 7.0 for bigger d Mol Biol Evol. 2016;33(7):1870-1874.

35. Krzywinski M, Schein JE, Biro I Circos: an information aesthetic for comparative genomics. Genome Res. 2009;19:1639-1645.

36. Yuan JP, Liu TK, Yu ZH, Li Y, Ren HB, Hou XL, et al. Genome-wide analysis of Chinese cabbage IQD gene family and the response of BrIQD5 in drought resistance. Plant Mol Biol. 2019;99:603-620.

37. Wang LQ, Guo K, Li Y, Tu YY, Hu HZ, Wang BR, et al. Expression profiling and integrative analysis of the CESA/CSL superfamily in rice. BMC Plant Biol. 2010;10:282-297.

38. Emanuelsson O, Nielsen H, Brunak S, Von HG. Predicting subcellular localization of proteins based on their N-terminal amino acid sequence. J Mol Biol. 2000;300:1005-1016.

39. Sun HH, Wu S, Zhang GY, Jiao C, Guo SG, Ren Y, et al. Karyotype stability and unbiased fractionation in the paleo-allotetraploid Cucurbita genomes. Mol Plant. 2017;10:1293-1306.

40. Zheng Y, Wu S, Bai Y, Sun HH, Jiao C, Guo SG, et al. Cucurbit Genomics Database (CuGenDB): a central portal for comparative and functional genomics of cucurbit crops. Nucleic Acids Res. 2019;47(1):1128-1136.

41. Livak KJ, Schmittgen TD. Analysis of relative gene expression data using realtime quantitative PCR and the $2^{-\triangle \Delta C T}$ method. Methods. 2001;25:402-408.

\section{Tables}

Table 1 Physical and chemical characteristics of the 34 YABBY genes identified in Cucurbita maxima, Cucurbita moschata and Cucurbita pepo. 


\begin{tabular}{|c|c|c|c|c|c|c|c|c|c|c|c|}
\hline Gene ID & Cultivar & Gene name & $\mathrm{Chr}^{\mathrm{a}}$ & Start ${ }^{b}$ & End $^{c}$ & $\begin{array}{l}\text { ORF } \\
\text { length } \\
\text { (bp) }\end{array}$ & pl & $\begin{array}{l}\text { Molecular } \\
\text { weight } \\
\text { (Da) }\end{array}$ & $\begin{array}{l}\text { Number } \\
\text { of } \\
\text { amino } \\
\text { acid } \\
\text { (aa) }\end{array}$ & $\begin{array}{l}\text { Subcellular } \\
\text { localization }\end{array}$ & $\begin{array}{l}\text { Aral } \\
\text { horr }\end{array}$ \\
\hline CmoCh04G017950.1 & $\begin{array}{l}\text { Cucurbita } \\
\text { moschata }\end{array}$ & CmoCRCa & Cmo_Chr04 & 9028899 & 9030980 & 606 & 9.08 & 22132.26 & 201 & Nucleus. & AT1 \\
\hline CmoCh18G011200.1 & $\begin{array}{l}\text { Cucurbita } \\
\text { moschata }\end{array}$ & $\mathrm{CmoCRCb}$ & Cmo_Chr18 & 11567244 & 11572287 & 708 & 9.99 & 25743.41 & 235 & Nucleus. & AT1 \\
\hline CmoCh05G000290.1 & $\begin{array}{l}\text { Cucurbita } \\
\text { moschata }\end{array}$ & CmolNOb & Cmo_Chr05 & 107469 & 108710 & 594 & 6.43 & 21846.84 & 197 & Nucleus. & AT1 \\
\hline CmoCh04G018270.1 & $\begin{array}{l}\text { Cucurbita } \\
\text { moschata }\end{array}$ & CmolNOa & Cmo_Chr04 & 9186167 & 9191600 & 930 & 9.35 & 35478.2 & 309 & Nucleus. & AT1 \\
\hline CmoCh02G015970.1 & $\begin{array}{l}\text { Cucurbita } \\
\text { moschata }\end{array}$ & CmoYAB1a & Cmo_Chr02 & 9202861 & 9204633 & 714 & 8.8 & 26238.55 & 237 & Nucleus. & AT2 \\
\hline CmoCh15G012090.1 & $\begin{array}{l}\text { Cucurbita } \\
\text { moschata }\end{array}$ & CmoYAB1b & Cmo_Chr15 & 8382484 & 8384352 & 711 & 8.8 & 26015.43 & 236 & Nucleus. & AT2 \\
\hline CmoCh02G013370.1 & $\begin{array}{l}\text { Cucurbita } \\
\text { moschata }\end{array}$ & CmoYAB2 & Cmo_Chr02 & 7963763 & 7967840 & 510 & 9.06 & 18889.49 & 169 & Nucleus. & AT1 \\
\hline CmoCh11G005350.1 & $\begin{array}{l}\text { Cucurbita } \\
\text { moschata }\end{array}$ & CmoYAB5c & Cmo_Chr11 & 2613770 & 2616030 & 573 & 7.56 & 21429.38 & 190 & Nucleus. & AT2 \\
\hline CmoCh05G013080.1 & $\begin{array}{l}\text { Cucurbita } \\
\text { moschata }\end{array}$ & CmoYAB5a & Cmo_Chr05 & 10151693 & 10155834 & 582 & 8.95 & 21726.63 & 193 & Nucleus. & AT2 \\
\hline CmoCh12G012310.1 & $\begin{array}{l}\text { Cucurbita } \\
\text { moschata }\end{array}$ & CmoYAB5d & Cmo_Chr12 & 10977446 & 10981728 & 570 & 8.98 & 21268.06 & 189 & Nucleus. & AT2 \\
\hline CmoCh10G005780.1 & $\begin{array}{l}\text { Cucurbita } \\
\text { moschata }\end{array}$ & CmoYAB5b & Cmo_Chr10 & 2633020 & 2635308 & 546 & 6.29 & 20280.73 & 181 & Nucleus. & AT2 \\
\hline CmaCh04G017090.1 & $\begin{array}{l}\text { Cucurbita } \\
\text { maxima }\end{array}$ & $\mathrm{CmaCRCa}$ & Cma_Chr04 & 8594676 & 8596684 & 537 & 8.81 & 19479.12 & 178 & Nucleus. & AT1 \\
\hline CmaCh18G010980.1 & $\begin{array}{l}\text { Cucurbita } \\
\text { maxima }\end{array}$ & $\mathrm{CmaCRCb}$ & Cma_Chr18 & 8991641 & 8995269 & 660 & 9 & 23653.51 & 219 & Nucleus. & AT1 \\
\hline CmaCh04G017410.1 & $\begin{array}{l}\text { Cucurbita } \\
\text { maxima }\end{array}$ & CmaINOa & Cma_Chr04 & 8746724 & 8752040 & 1179 & 8.19 & 43636.39 & 392 & Nucleus. & AT1 \\
\hline CmaCh05G000220.1 & $\begin{array}{l}\text { Cucurbita } \\
\text { maxima }\end{array}$ & CmalNOb & Cma_Chr05 & 105423 & 106388 & 576 & 6.37 & 21366.42 & 191 & Nucleus. & AT1 \\
\hline CmaCh02G015570.1 & $\begin{array}{l}\text { Cucurbita } \\
\text { maxima }\end{array}$ & CmaYAB1a & Cma_Chr02 & 8833805 & 8835686 & 696 & 8.8 & 25570.87 & 231 & Nucleus. & AT2 \\
\hline CmaCh15G011490.1 & $\begin{array}{l}\text { Cucurbita } \\
\text { maxima }\end{array}$ & CmaYAB1b & Cma_Chr15 & 7308199 & 7310013 & 711 & 8.8 & 25929.29 & 236 & Nucleus. & AT2 \\
\hline CmaCh02G012970.1 & $\begin{array}{l}\text { Cucurbita } \\
\text { maxima }\end{array}$ & CmaYAB2 & Cma_Chr02 & 7630829 & 7634766 & 540 & 9.32 & 19978.84 & 179 & Nucleus. & AT1 \\
\hline CmaCh11G005360.1 & $\begin{array}{l}\text { Cucurbita } \\
\text { maxima }\end{array}$ & CmaYAB5c & Cma_Chr11 & 2583470 & 2590191 & 681 & 8.06 & 25346.08 & 226 & Nucleus. & AT2 \\
\hline CmaCh05G012870.1 & $\begin{array}{l}\text { Cucurbita } \\
\text { maxima }\end{array}$ & CmaYAB5a & Cma_Chr05 & 9820965 & 9824076 & 582 & 8.95 & 21715.6 & 193 & Nucleus. & AT2 \\
\hline CmaCh12G012020.1 & $\begin{array}{l}\text { Cucurbita } \\
\text { maxima }\end{array}$ & CmaYAB5d & Cma_Chr12 & 9427635 & 9431144 & 570 & 8.86 & 21179.89 & 189 & Nucleus. & AT2 \\
\hline CmaCh10G005410.1 & $\begin{array}{l}\text { Cucurbita } \\
\text { maxima }\end{array}$ & CmaYAB5b & Cma_Chr10 & 2483897 & 2486274 & 537 & 6.8 & 19988.49 & 178 & Nucleus. & AT2 \\
\hline CmaCh06G007630.1 & $\begin{array}{l}\text { Cucurbita } \\
\text { maxima }\end{array}$ & CmaYAB3 & Cma_Chr06 & 3875334 & 3877797 & 606 & 8.61 & 22196.04 & 201 & Nucleus. & AT4 \\
\hline Cp4.1LG09g02290.1 & $\begin{array}{l}\text { Cucurbita } \\
\text { pepo }\end{array}$ & CpeCRC & Cp4.1LG09 & 1313947 & 1316378 & 498 & 6.63 & 17647.61 & 165 & Nucleus. & AT1 \\
\hline Cp4.1LG01g15370.1 & $\begin{array}{l}\text { Cucurbita } \\
\text { pepo }\end{array}$ & CpelNOa & Cp4.1LG01 & 9207257 & 9210078 & 573 & 8.57 & 21514.93 & 190 & Nucleus. & AT1 \\
\hline Cp4.1LG11g00620.1 & $\begin{array}{l}\text { Cucurbita } \\
\text { pepo }\end{array}$ & CpelNOb & Cp4.1LG11 & 100544 & 102144 & 471 & 9.3 & 17044.70 & 156 & Nucleus. & AT1 \\
\hline Cp4.1LG13g02080.1 & $\begin{array}{l}\text { Cucurbita } \\
\text { pepo }\end{array}$ & CpeYAB1b & Cp4.1LG13 & 1656187 & 1659056 & 639 & 9.76 & 23823.32 & 212 & Nucleus. & AT2 \\
\hline
\end{tabular}




\begin{tabular}{|c|c|c|c|c|c|c|c|c|c|c|c|}
\hline Cp4.1LG05g02370.1 & $\begin{array}{l}\text { Cucurbita } \\
\text { pepo }\end{array}$ & CpeYAB1a & Cp4.1LG05 & 1340984 & 1343955 & 1110 & 9.37 & 40999.44 & 369 & Nucleus. & AT2 \\
\hline Cp4.1LG05g04630.1 & $\begin{array}{l}\text { Cucurbita } \\
\text { pepo }\end{array}$ & CpeYAB2 & Cp4.1LG05 & 2635623 & 2639712 & 510 & 9.21 & 18817.42 & 169 & Nucleus. & AT1 \\
\hline Cp4.1LG11g10670.1 & $\begin{array}{l}\text { Cucurbita } \\
\text { pepo }\end{array}$ & CpeYAB5c & Cp4.1LG11 & 8932872 & 8936484 & 582 & 8.94 & 21738.64 & 193 & Nucleus. & AT2 \\
\hline Cp4.1LG07g11420.1 & $\begin{array}{l}\text { Cucurbita } \\
\text { pepo }\end{array}$ & CpeYAB5b & Cp4.1LG07 & 9727251 & 9734543 & 582 & 8.86 & 21686.53 & 193 & Nucleus. & AT2 \\
\hline Cp4.1LG18g04950.1 & $\begin{array}{l}\text { Cucurbita } \\
\text { pepo }\end{array}$ & CpeYAB5d & Cp4.1LG18 & 5778295 & 5780743 & 495 & 6.7 & 18255.67 & 164 & Nucleus. & AT2 \\
\hline Cp4.1LG04g09420.1 & $\begin{array}{l}\text { Cucurbita } \\
\text { pepo }\end{array}$ & CpeYAB5a & Cp4.1LG04 & 10087072 & 10089482 & 519 & 6.06 & 19308.98 & 172 & Nucleus. & AT2 \\
\hline Cp4.1LG08g07580.1 & $\begin{array}{l}\text { Cucurbita } \\
\text { pepo }\end{array}$ & CpeYAB3 & Cp4.1LG08 & 6069752 & 6071866 & 579 & 8.6 & 21203.08 & 192 & Nucleus. & AT4 \\
\hline
\end{tabular}

a The chromosome in which the gene is located

b The start position of the gene on the chromosome

c The end position of the gene on the chromosom

Table $2 \mathrm{Ka} / \mathrm{Ks}$ calculation and estimated divergence time for the $Y A B B Y$ duplicated gene pairs

\begin{tabular}{|c|c|c|c|c|c|c|c|c|c|}
\hline Gene ID & $\begin{array}{l}\text { Identity } \\
(\%)\end{array}$ & $\begin{array}{l}\text { Alignment } \\
\text { length } \\
\text { (bp) }\end{array}$ & $\begin{array}{l}\text { Mismatches } \\
\text { (bp) }\end{array}$ & $\begin{array}{l}\text { length of the larger } \\
\text { gene } \\
\text { (bp) }\end{array}$ & $\begin{array}{l}\text { Gene alignment } \\
\text { coverage }\end{array}$ & $\mathrm{Ka}$ & Ks & $\mathrm{Ka} / \mathrm{Ks}$ & $\begin{array}{l}\text { Divergence } \\
\text { time } \\
\text { (MYA) }\end{array}$ \\
\hline $\begin{array}{l}\text { CmoYAB1a- } \\
\text { CmoYAB1b }\end{array}$ & 84.92 & 723 & 68 & 714 & 0.92 & 0.06 & 0.42 & 0.14 & 14.12 \\
\hline $\begin{array}{l}\text { CmoYAB5a- } \\
\text { CmoYAB5d }\end{array}$ & 89.71 & 583 & 46 & 582 & 0.92 & 0.06 & 0.22 & 0.28 & 7.26 \\
\hline $\begin{array}{l}\text { CmoYAB5c- } \\
\text { CmoYAB5b }\end{array}$ & 85.58 & 541 & 57 & 573 & 0.84 & 0.10 & 0.49 & 0.20 & 16.47 \\
\hline $\begin{array}{l}\text { CmaYAB1b- } \\
\text { CmaYAB1a }\end{array}$ & 87.04 & 702 & 60 & 711 & 0.90 & 0.04 & 0.32 & 0.13 & 10.72 \\
\hline $\begin{array}{l}\text { CmaYAB5d- } \\
\text { CmaYAB5a }\end{array}$ & 90.38 & 582 & 44 & 582 & 0.92 & 0.06 & 0.20 & 0.30 & 6.64 \\
\hline $\begin{array}{l}\text { CpeYAB5b- } \\
\text { CpeYAB5c }\end{array}$ & 89.71 & 583 & 46 & 582 & 0.92 & 0.06 & 0.24 & 0.23 & 8.15 \\
\hline
\end{tabular}

\section{Figures}




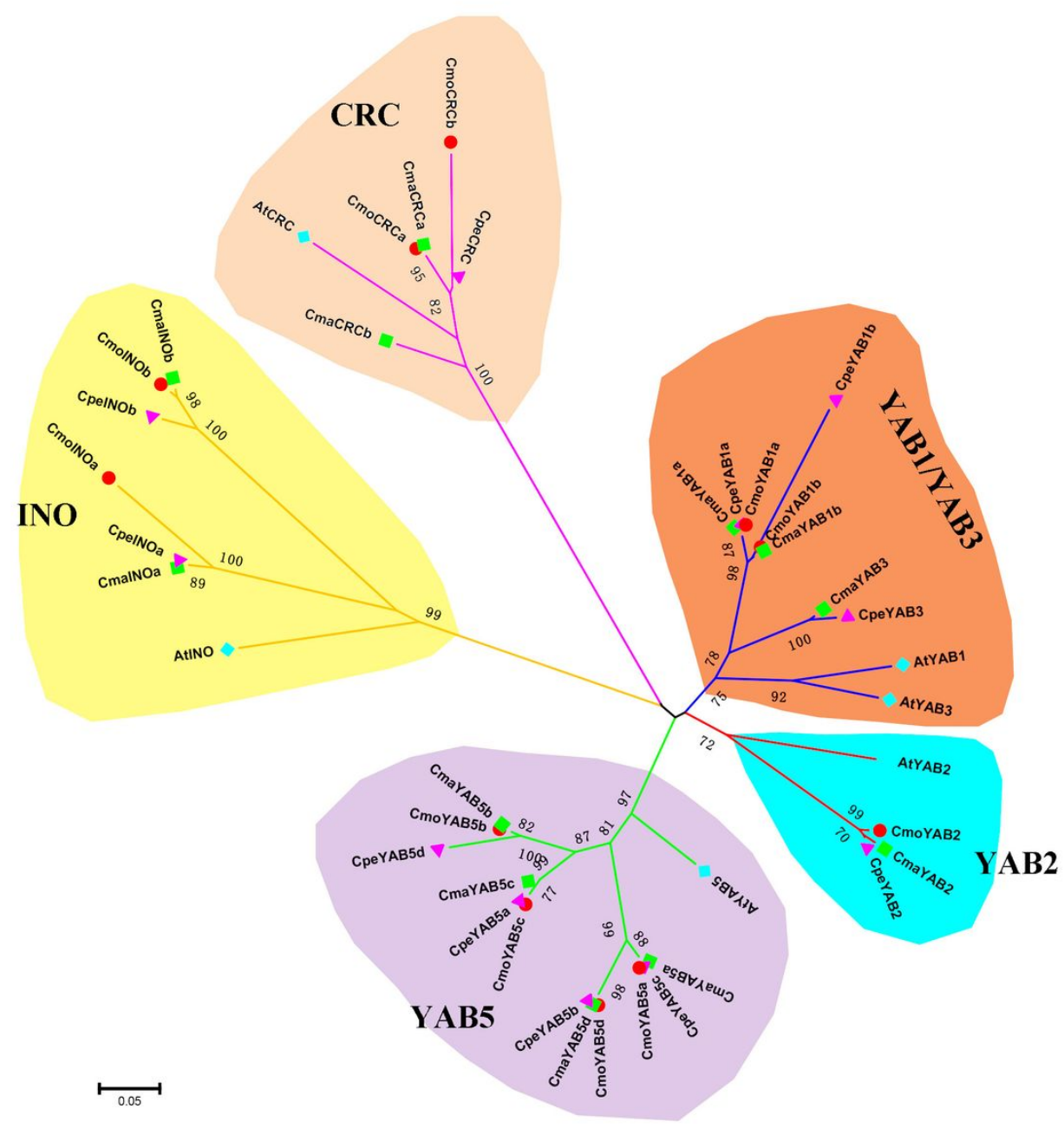

\section{Figure 1}

Phylogenetic trees of the YABBY gene family in Cucurbita moschata, Cucurbita maxima, Cucurbita pepo and Arabidopsis thaliana. Five different subfamilies (YAB1/YAB3, YAB2, INO, CRC, and YAB5) were displayed with different background colors. 
A

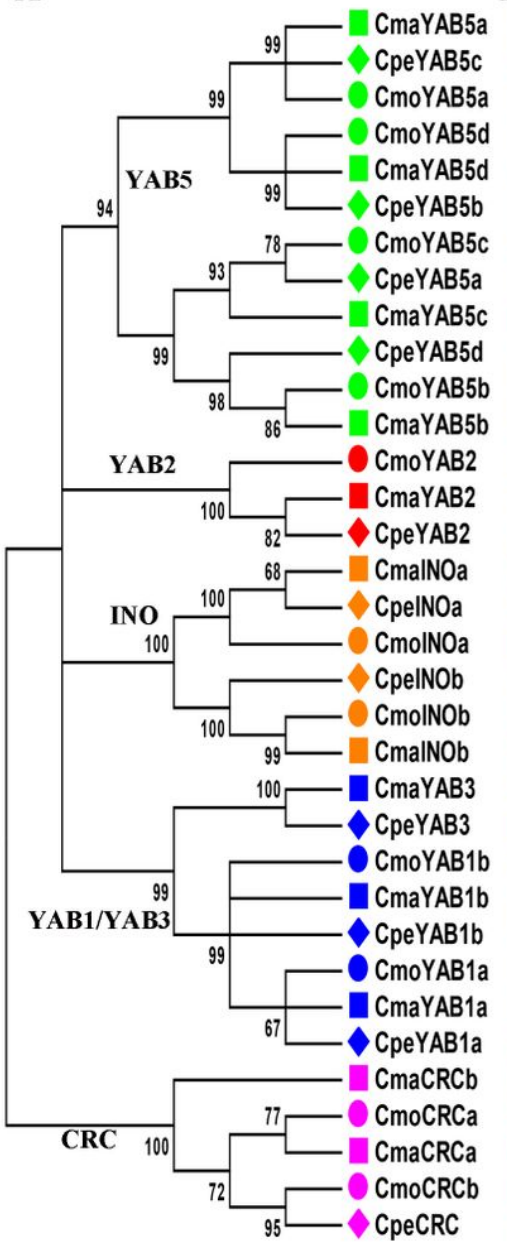

B

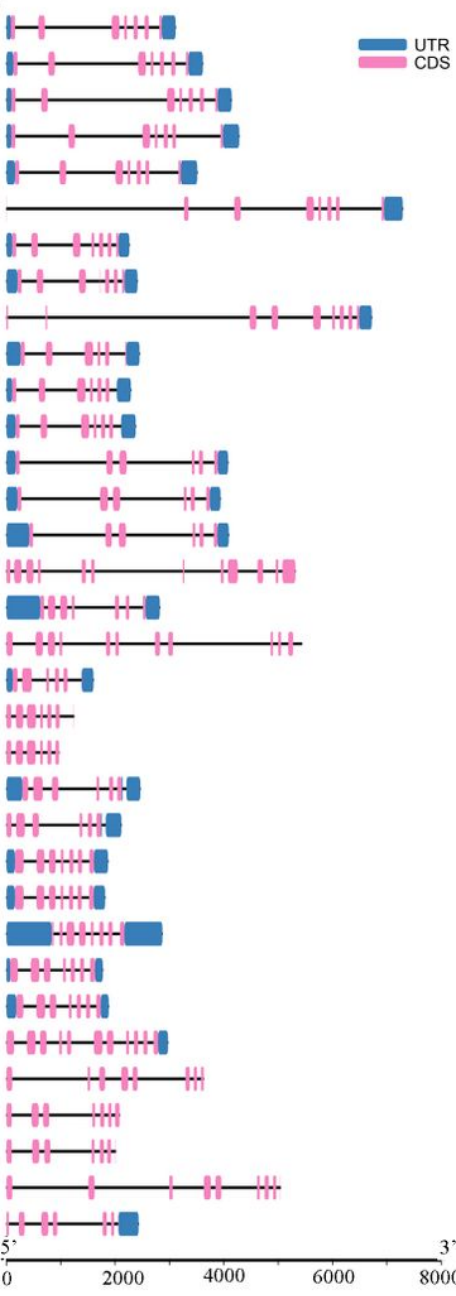

C

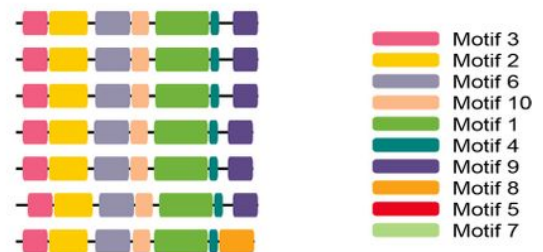

\section{Figure 2}

Gene structure and motif composition of YABBYs gene family in three Cucurbita Linn. cultivars. A. The phylogenetic tree of 34 YABBYs was constructed using the neighbor-joining $(\mathrm{NJ})$ method with 1,000 bootstrap replicates, and a $65 \%$ cut-off value was used for the condensed tree. B. Exon-intron organization of 34 YABBYs in three cultivars of Cucurbita Linn.. The exons and introns were represented by pink boxes and black lines, respectively. Untranslated region (UTR) were indicated by blue boxes. The sizes of the exons and introns can be estimated using the scale at the bottom. C. Schematic representation of conserved motifs in 34 YABBYs. Each motif was represented by a numbered coloured box on the right. The same number in different proteins refers to the same motif. 
A

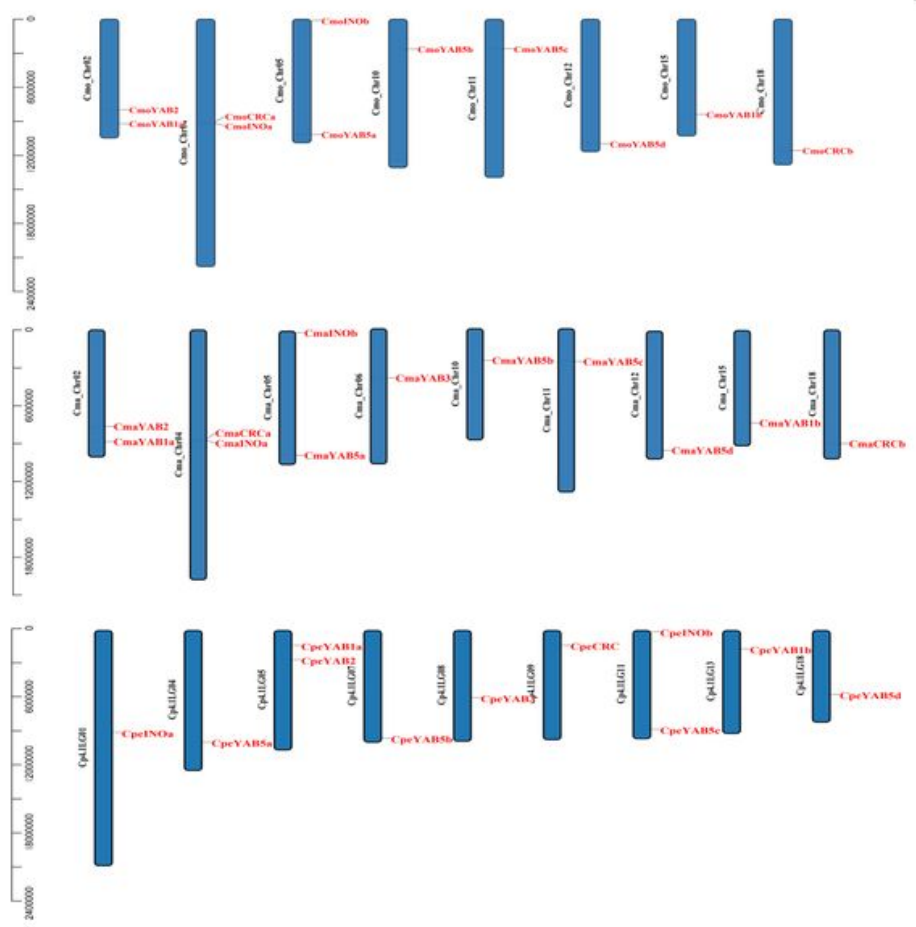

B

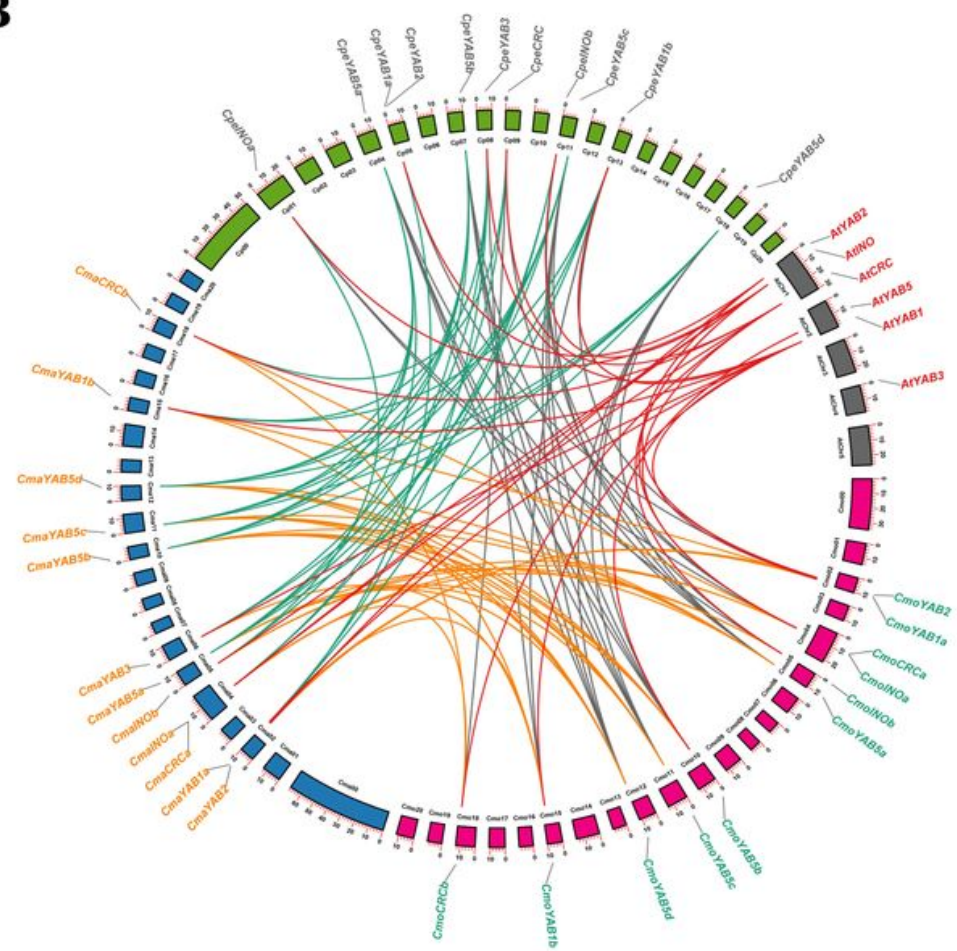

Figure 3

Distribution of YABBY family genes in three cultivars of Cucurbita Linn. and the collinear relationship between Cucurbita Linn. YABBY genes and AtYABBY genes. A. The distribution of YABBY genes from Cucurbita moschata, Cucurbita maxima, and Cucurbita pepo on a chromosome. B. Among the YABBY genes of Cucurbita moschata, Cucurbita maxima and Cucurbita pepo, the collinear genes of AtYABBY (represented by red lines), and the collinear genes between Cucurbita moschata, Cucurbita maxima, and Cucurbita pepo. The green lines indicated the collinearity between the CmaYABBY gene and the CpeYABBY gene; the orange lines indicated the collinearity between the CmaYABBY gene and CmoYABBY gene; the gray lines indicated the collinearity between CmoYABBY gene and CpeYABBY gene.

A

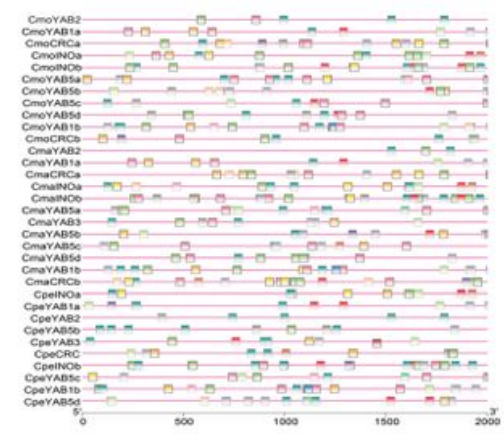

C

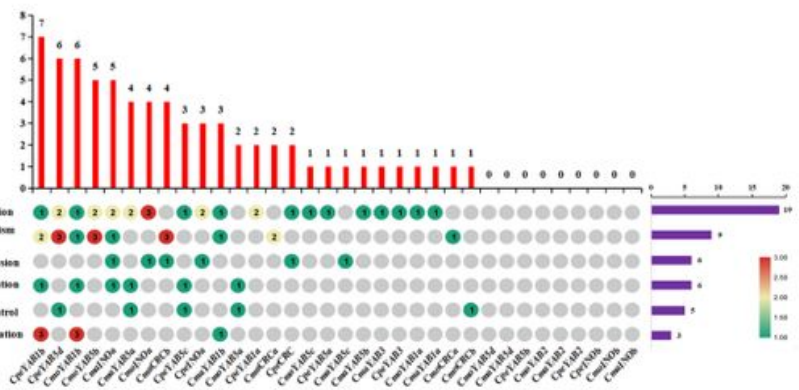

B
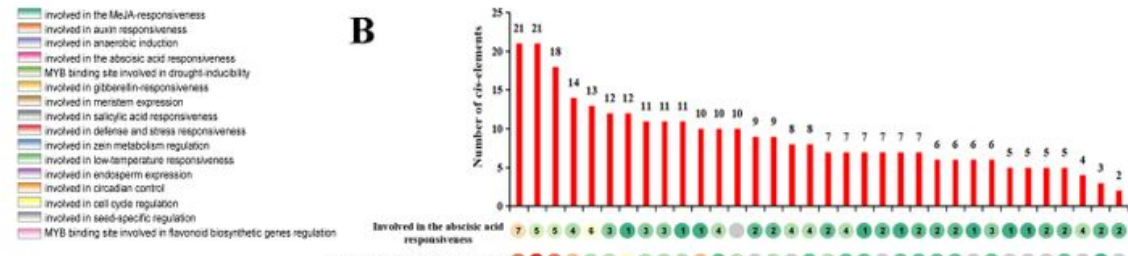

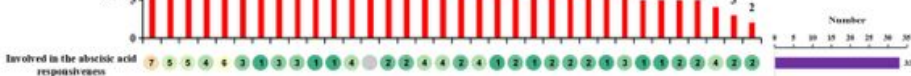

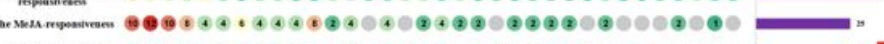

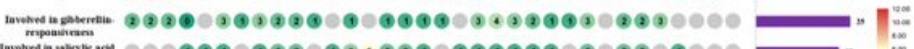

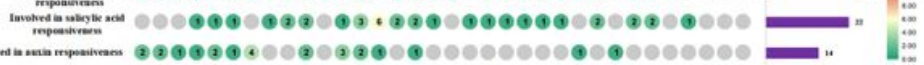

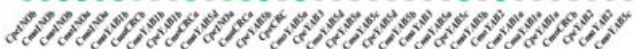

D

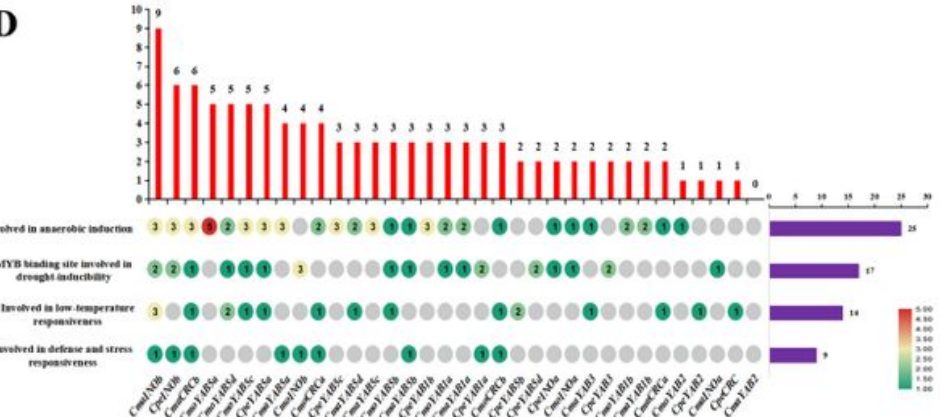

Figure 4

Distribution and statistics of cis-acting elements of YABBY gene promoter in three Cucurbitaceae cultivars. A, The schematic diagram of the cis-acting elements of the 34 YABBY gene promoters; B, Statistical analysis of the cis-acting elements of 34 YABBY genes involved in hormone response; C, Statistical 
analysis of the cis-acting elements of 34 YABBY genes involved in plant growth and development; D, Statistical analysis of the cis-acting elements of 34 YABBY genes involved in abiotic stress response.

A

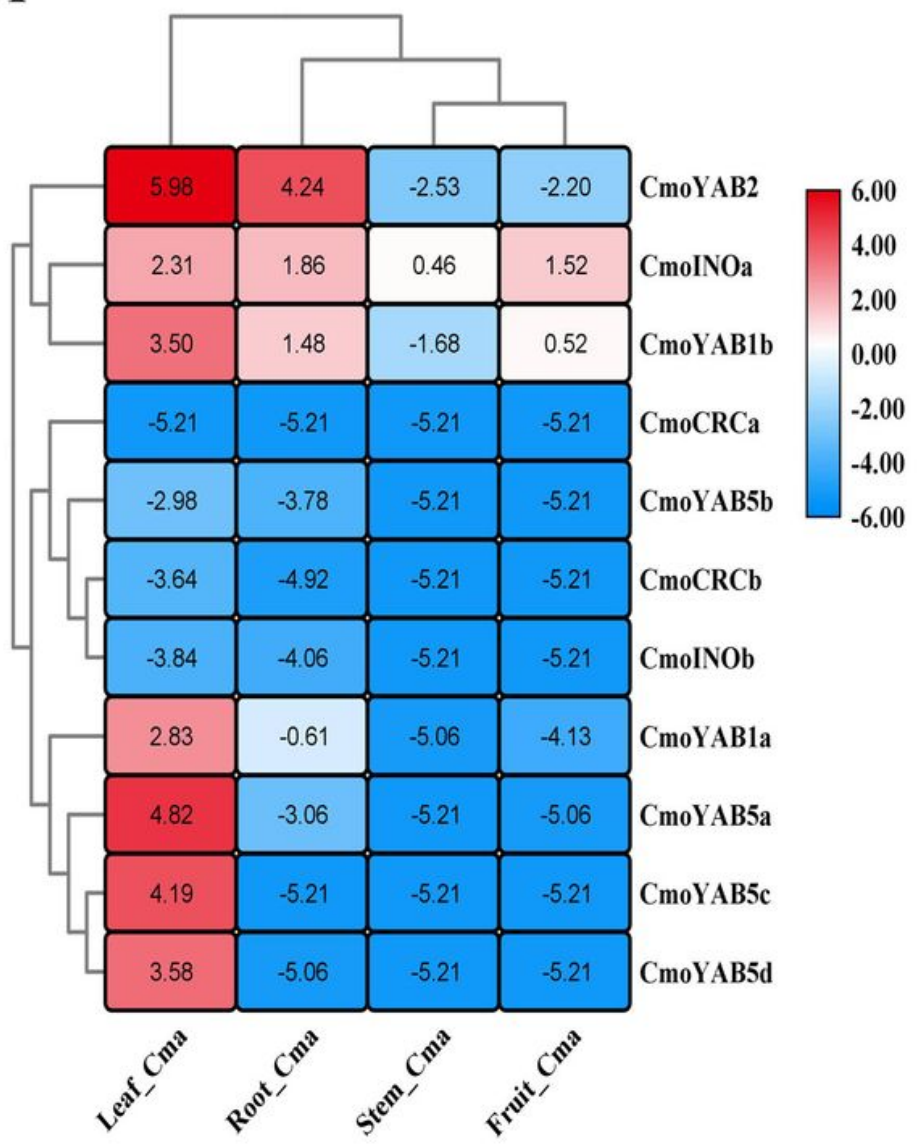

B

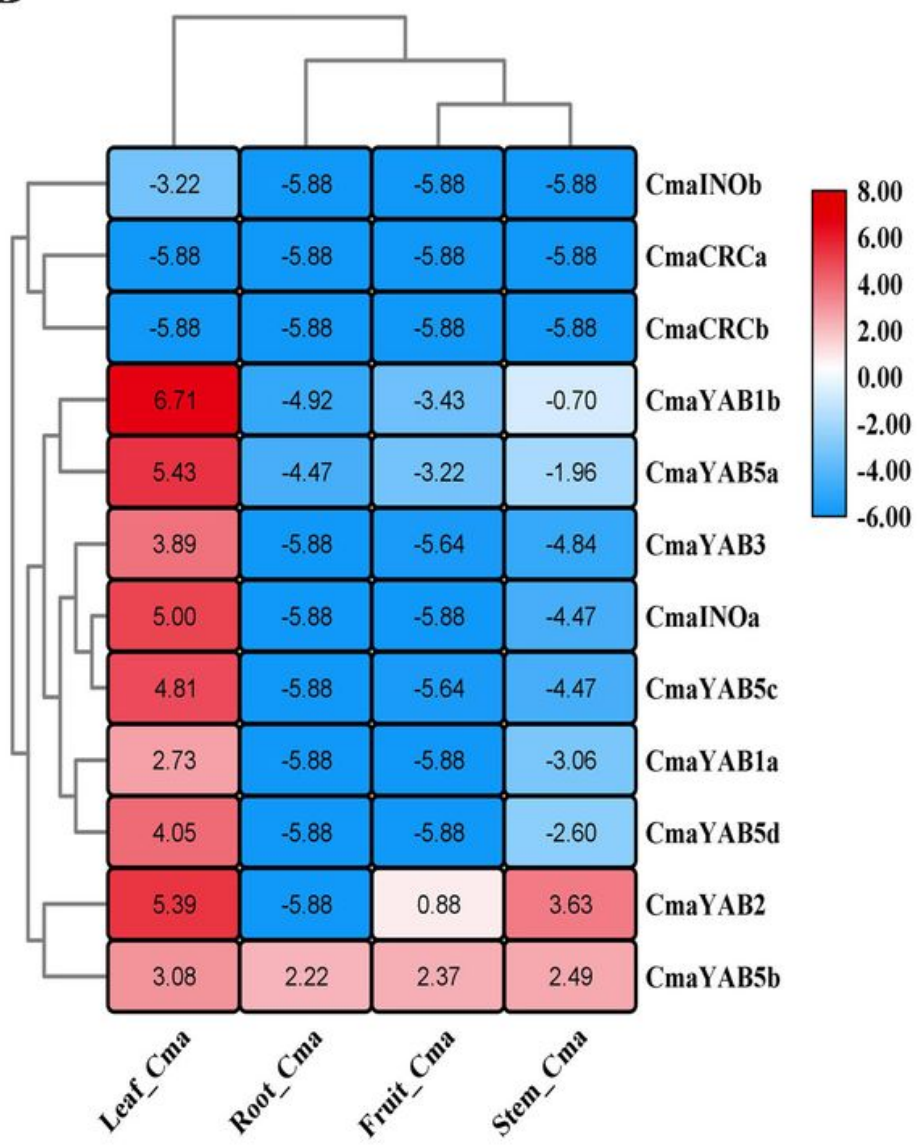

Figure 5

The relative expression levels of CmoYABBYs and CmaYABBYs in roots, stems, leaves, and fruits. A. The relative expression levels and clustering of CmoYABBYs in roots, stems, leaves, and fruits; B, The relative expression levels and clustering of CmaYABBYs in roots, stems, leaves, and fruits. The bar at the right of the heat map represented the relative expression values. The corresponding expression level was expressed as log2 (RPKM). 


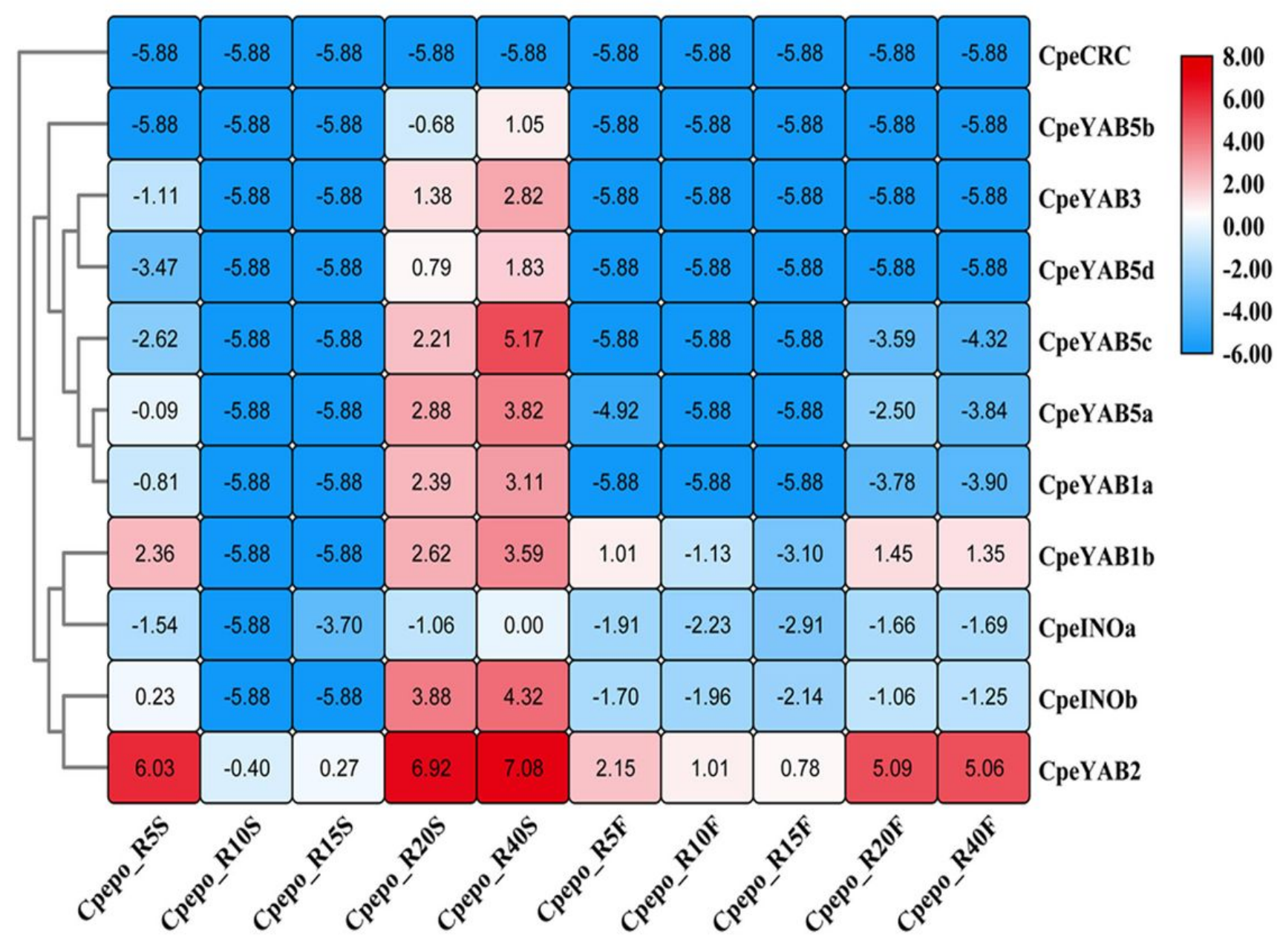

Figure 6

The relative expression levels and clustering of CpeYABBYs from "Sweet REBA" in seed and fruit mesocarp. The bar at the right of the heat map represented the relative expression values. The corresponding expression level was expressed as log2 (RPKM). R: Sweet REBA; S: seed; F: fruit mesocarp. 5, 10, 15, 20 , 40: 5 DAP , 10 DAP, 15 DAP, 20 DAP, 40 DAP; DAP, days after pollination. 


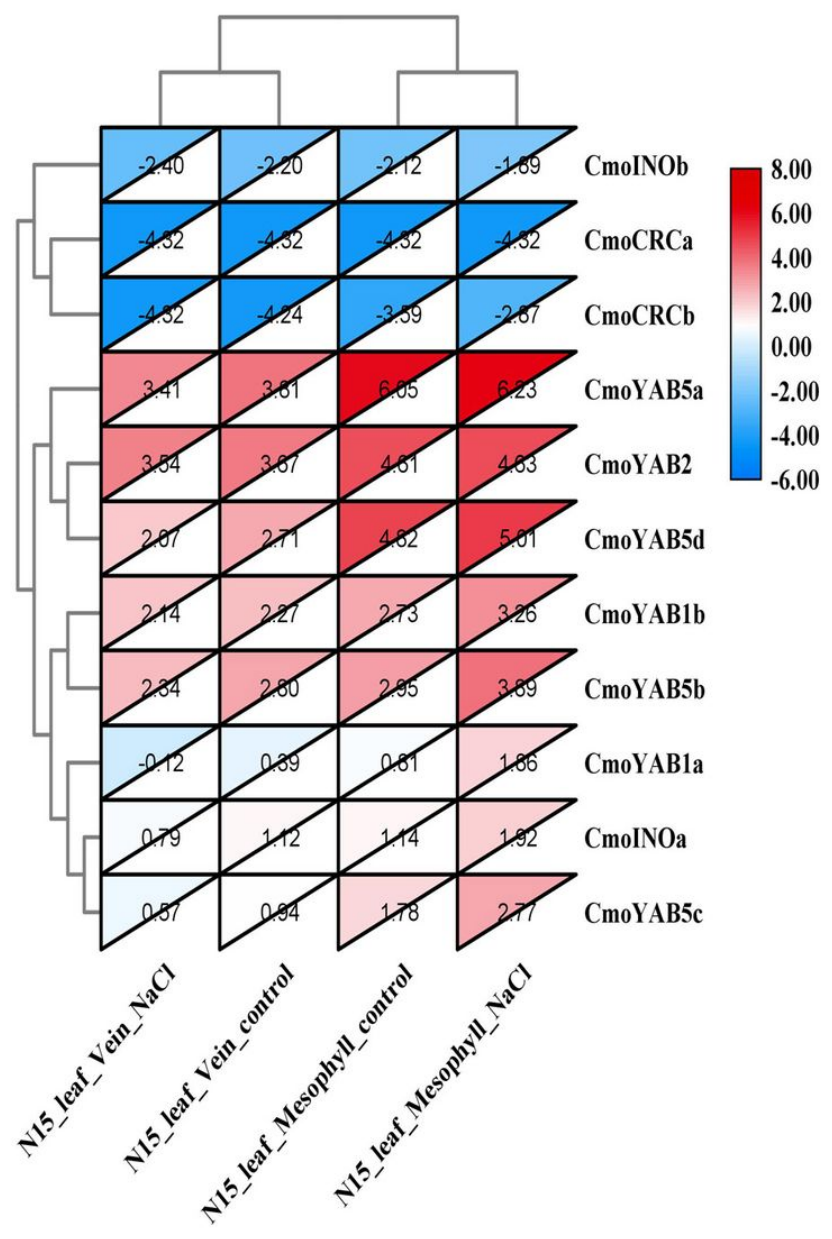

Figure 7

The relative expression level of the YABBY gene in leaf mesophyll and leaf vein of Cucurbita moschata under salt stress. The heatmap and cluster showed the relative expression level and clustering of CmoYABBY genes under $\mathrm{NaCl}$ treatment and control treatments. The bar at the right of the heat map represented the relative expression values. The corresponding expression level was expressed as log2 (RPKM). N15: Cucurbita moschata ("Rimu"). 


\begin{tabular}{|c|c|c|}
\hline 0.08 & 0.55 & CmoCRCa \\
\hline 0.02 & 16.23 & CmoCRCb \\
\hline 4.76 & 30.72 & CmoINOb \\
\hline 0.02 & 0.52 & CmoINOa \\
\hline 0.02 & 0.07 & CmoYAB1a \\
\hline 0.03 & 0.04 & CmoYAB1b \\
\hline 0.05 & 0.33 & CmoYAB2 \\
\hline 0.02 & 0.12 & CmoYAB5c \\
\hline 0.02 & 0.22 & CmoYAB5a \\
\hline 0.04 & 3.46 & CmoYAB5d \\
\hline 6.00 & 1.73 & CmoYAB5b \\
\hline
\end{tabular}

Figure 8

Heat map of the YABBY gene in Cucurbita moschata ("Baimi 9 ") in root tip under salt stress. The results were calculated via the $2-\Delta \Delta C t$ method, and the reference gene ( $\beta$-Actin) was used to correct the expression level of target genes.

\section{Supplementary Files}

This is a list of supplementary files associated with this preprint. Click to download.

- Fig.S1.tif

- Fig.S2.tif

- Tables1.docx

- Tables2.docx 